\title{
A solar eruption triggered by merge interactions of different helicity loops
}

\author{
W. Song, X. Feng, and F. Shen \\ SIGMA Weather Group, State Key Laboratory for Space Weather, Center for Space Science and Applied Research, Chinese Academy \\ of Sciences, Beijing 100190, PR China \\ e-mail: [wbsong; fengx; fshen] @spaceweather .ac.cn \\ Received 20 March 2008 / Accepted 9 May 2008 \\ ABSTRACT

\begin{abstract}
Aims. We investigate the magnetic evolution of a flare/CME source region to determine the trigger of the flare and the EUV brightening event. Also, we discuss the role of the current helicity $\left(h_{\mathrm{c}}\right)$ in solar activity.

Methods. We use the long duration sequences of SOHO/MDI magnetograms and TRACE $195 \AA$ images for a super active region (AR), NOAA AR 8375. Magnetic field changes in the photosphere and the corona are investigated.

Results. In AR 8375, the southwestern and northwestern parts of an overlying loop $\left(h_{\mathrm{c}}<0\right)$ are influenced by the rising magnetic arcades $\left(h_{\mathrm{c}}>0\right)$ and the emerging flux region $\left(h_{\mathrm{c}}>0\right)$. Two collisions make the overlying loop inflate in a bounce interaction. However, the related solar events are triggered by a merge interaction which takes place among the fibers of the rising magnetic arcades and among those of the overlying loop.
\end{abstract}

Key words. Sun: flares - Sun: coronal mass ejections (CMEs) - Sun: magnetic fields

\section{Introduction}

In numerous investigations (e.g., Nitta \& Hudson 2001; Pohjolainen 2003; Moon et al. 2004), a very large monopolar sunspot with persistent flux appearance has been found to trigger many solar events. For the active region of AR 9236 and a group of flares and coronal mass ejections (CMEs), Nitta \& Hudson (2001) attribute the triggering of solar eruptions to new emerging flux, while Zhang \& Wang (2002) propose that the moving magnetic features (MMFs) also play a role since the MMFs show emergence of small-scale flux. For another AR (AR 8375), Yurchyshyn \& Wang (2001) find a possible connection between the flare and the sunspot moat flux cancellation. Romano et al. (2003) report a strong temporal correlation between the filament eruption and the helicity transport from the photospheric magnetic structures at the filament foot points into the corona. These authors mainly investigate the variability of the photosphere. These different viewpoints indicate that the actual triggering mechanism is still uncertain. To better determine what triggers the flare/CME events, one should also examine the corona. In this letter we revisit AR 8375 and one of its related flares. AR 8375 has a simple configuration whose flux evolution can be followed without ambiguity. There are two time sequences taken with high resolution and in high cadence mode, one of the photosphere (the magnetograms obtained with the Michelson Doppler Imager (MDI) on board the Solar and Heliospheric Observatory (SOHO)), the other is of the corona (the EUV images taken by Transition Region and Coronal Explorer (TRACE)). The two sequences are examined simultaneously.

\section{A C5.2 flare and a north-directed CME}

A C-class flare and a north-directed CME with an angular width of $69^{\circ}$ were observed to occur in AR 8375 by four satellites.
As shown in Fig. 1, (a) the plot of GOES X-ray flux indicates that there is a C5.2 flare that erupted at 03:10 UT and reached its maximum at 03:35 UT; (b) at 03:28 UT, in the flare source region of AR 8375, TRACE observed distinct flaring arcades (FA) in the southwestern corner; (c) from the soft X-ray movie taken by $\mathrm{YOHKOH}$, near the time of flare maximum, we find a north-directed ejection overlapping the flare source region; (d) the difference image in SOHO/LASCO C2 confirms the existence of a north-directed $\mathrm{CME}$ caught by $\mathrm{YOHKOH}$. This is also the only north-directed CME listed in the SOHO LASCO CME CATALOG on November 4, 1998.

To compare TRACE $195 \AA$ images and SOHO/MDI magnetograms, we shrink, move and rotate the TRACE images so that they have the same pixel resolution and field of view as MDI. While adjusting the TRACE images, we determined a reference configuration. As shown in Figs. $2 a-d$, the outlines of EUV bright regions correlate well with those of MDI strong negative regions. From Figs. 2c, d we find two negative foot points of FA close together and two positive foot points, one in the umbra, the other on the sunspot outskirts (see four arrows). An EUV brightening is detected by TRACE at position "F2" near the flare maximum. The small two-peaked structure found in the C5.2 flare peak at 03:20 UT and 03:35 UT in Fig. 1a is possibly due to a later brightening event. However, with the different mechanisms of X-ray and EUV emission, the two-peaked structure that occurred in X-ray flux may also be related to the flare's own evolution. In the MDI movie, between "F2" and the umbra, we find an emerging flux region (EFR) which first appears in the sunspot moat on November 3 at 19:30 UT. Its two polarities move outward along helical paths in different directions (see the circles and short curves): negative flux moves counterclockwise, while positive flux moves clockwise. Their average radial (leaving the sunspot) and separation (circling around the sunspot) velocities are both near $0.3 \mathrm{~km} \mathrm{~s}^{-1}$. 

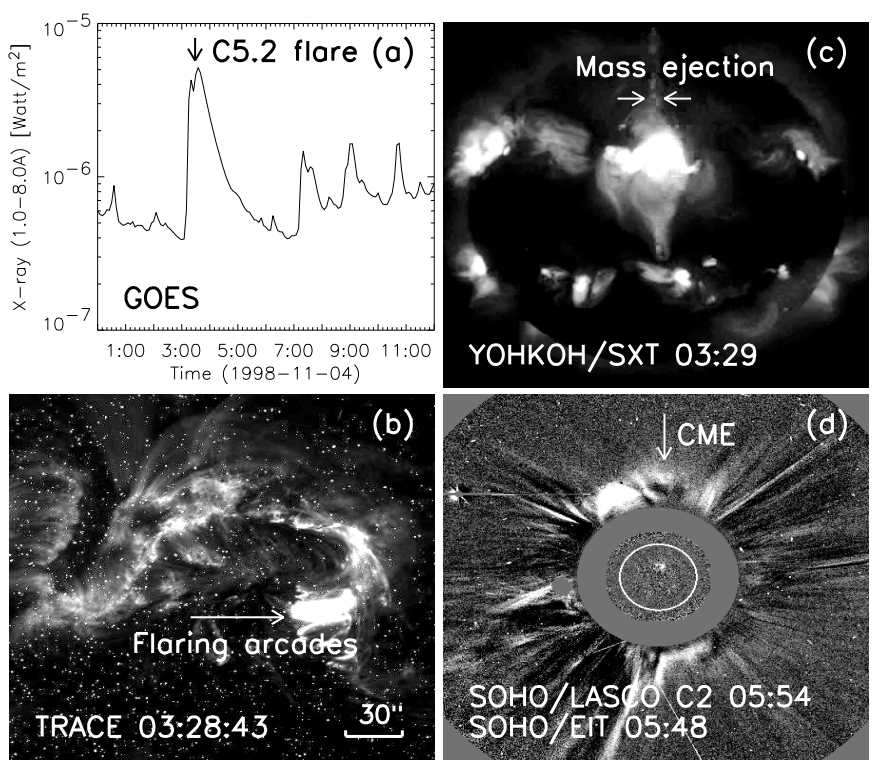

Fig. 1. a) Plot of the GOES X-ray (1.0-8.0 A) flux. A C5.2 flare occurs at 03:10 UT; b) TRACE reveals the flaring arcades at $195 \AA$; c) near the time of flare maximum, YOHKOH/SXT records a distinct northdirected mass ejection; d) several hours later, SOHO/LASCO C2 observes such ejection in its field of view.
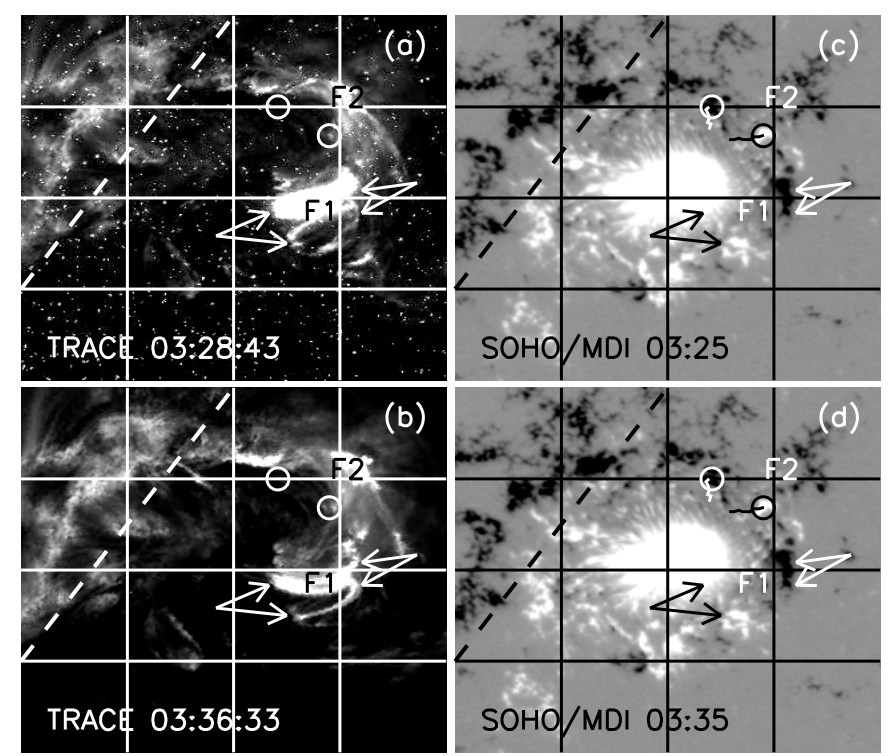

Fig. 2. The comparison between TRACE $195 \AA$ images (left, after being shrunk, moved and rotated) and SOHO/MDI magnetograms (right). MDI magnetograms are scaled from $-500 \mathrm{G}$ to $500 \mathrm{G}$. "F1" and "F2" indicate the C5.2 flare and the EUV brightening event. Four arrows mark the foot points of the "F1" flaring arcades. Circles mark the emerging flux region (EFR) around which two short curves depict the EFR's trajectory.

\section{Magnetic evolution of the flare/CME source region}

In Figs. 3a, b, several minutes before the flare eruption, we find an inflating flux loop located above the FA. Both ends of the loop extend much farther than those of the FA. Here we call it the overlying loop (OL). Two arrows mark the OL's inner (Fig. 3a)
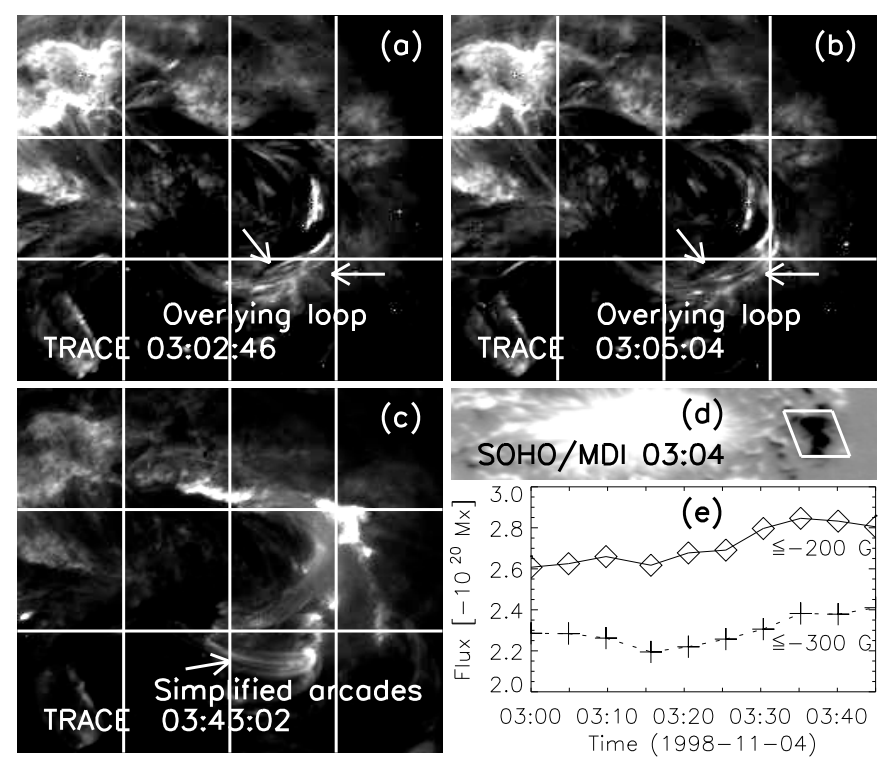

Fig. 3. a), b) Several minutes before the C5.2 flare, at position "F1", TRACE observes the overlying loop inflating rapidly; c) after the flare, the flaring arcades (FA) become simpler; d) SOHO/MDI magnetogram. The white polygon marks the FA's negative foot points; e) plot of the total magnetic flux in the region of the polygon in Fig. 3d. The solid line includes pixels with values $\leq-200 \mathrm{G}$, the dashed line value $\leq-300 \mathrm{G}$.

and outer (Fig. 3b) borders. They show that the OL's southwestern part rapidly inflated in less than three minutes. We estimate the average inflation velocity to be as high as $19.5-25.8 \mathrm{~km} \mathrm{~s}^{-1}$. In Fig. 3c, after the "F1" flare the FA becomes much thinner and more simplified. This is possibly due to the north-directed CME removing the magnetic field and matter.

During the flaring time, MDI magnetograms show no distinct variability. The FA's three foot points (the one immersed in the umbra is excluded) move as slowly as the EFR does. In Fig. 3d we draw the outlines of the FA's negative foot points and calculate the total magnetic flux in such a region. The time evolution of the total magnetic flux is shown in Fig. 3e in which the solid line includes pixels with a value lower than -200 Gauss, while the dashed line represents values lower than -300 Gauss. Both curves show a slow increase in the total magnetic flux. Therefore it is difficult to judge what causes the FA to rise, the foot points move, the magnetic emergence, etc.

In Figs. 4a, b, the EUV brightening at "F2" first takes place above the EFR. Then as shown in Figs. 4c, d, the brightening spreads along a large magnetic loop whose foot points and shape are very similar to those of the OL in Figs. 3a, b. We think that such a loop and the OL are the same structure, which involves slight expansion in the northwestern part. In addition, from Figs. 4a, b, we can detect clearly the unbrightened interface between the EFR and the OL so that the brightening energy should not be released from the interface.

Thus, the southwestern and northwestern parts of the OL are impacted by the FA and the EFR successively. They result in a C5.2 flare and an EUV brightening event. According to the positions of the OL, the FA and the EFR, we demonstrate the detailed magnetic configuration in Fig. 5. The white arc indicates the OL, the black arcs indicate the FA and the EFR, the color background indicates the contour of current helicity $\left(h_{\mathrm{c}}\right)$, and the arrowheads indicate the magnetic field directions. Here the directions of axial magnetic fields can be easily specified 


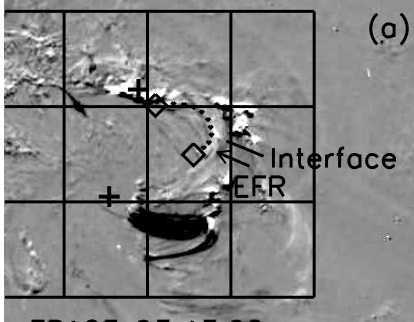

TRACE 03:43:02

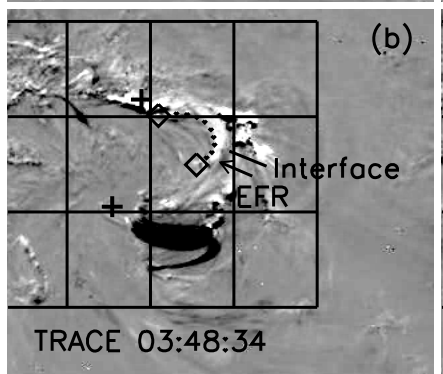

(b)

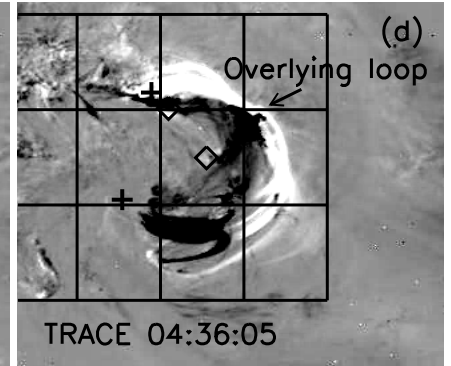

Fig. 4. Sequence of TRACE $195 \AA$ images subtracted from a reference image at 03:36:33 UT (see Fig. 2b). a), b) the EUV brightening happens near the interface between the emerging flux region (EFR) and the overlying loop $(\mathrm{OL}) ; \mathbf{c}), \mathbf{d})$ the whole OL is indicated. " $\diamond$ " and "+" indicate the foot points of the EFR and the OL. The black grids indicate the positions of the field of view of Figs. $3 a-c$.

from MDI magnetograms. However to find the directions of azimuthal magnetic fields, we must use the $h_{\mathrm{c}}$ distribution. From the arc foot points in Fig. 5, we find the $h_{\mathrm{c}}$ of OL, FA and EFR to be "-", "+" and "+", respectively. Then using the formula $h_{\mathrm{c}}=\mathbf{B} \cdot(\nabla \times \mathbf{B})$, we can work out their azimuthal magnetic field directions (see the arrowheads on the blue ellipses). In this figure, at both "F1" and "F2" interfaces, the magnetic fields of two colliding flux systems including the axial and the azimuthal components are almost parallel.

\section{Discussion}

Wang et al. (2004) suggested that the C-class flare that occurred in AR 8375 was triggered by the interaction between MMFs and the enhanced negative flux region (or the FA's negative foot points). Other studies attribute the triggering factor to the flux cancellation and helicity transport (Yurchyshyn \& Wang 2001; Romano et al. 2003). However, few of them give a definite model to discuss how their factors operate, especially during the process in the corona where solar events take place. Using MDI and TRACE data, we find the flare to be triggered by the rising FA, and the EUV brightening by the EFR. The question of what makes the FA rise, the flux cancellation, the interaction related to MMFs, etc, cannot be resolved here.

Linton et al. (2001) perform a 3D simulation to explore the physics of the collision of pairs of twisted flux tubes. In Fig. 5, the magnetic configurations at both "F1" and "F2" positions accord well with Linton's case RL0 ("R" - positive $h_{\mathrm{c}}$, "L" - negative $h_{\mathrm{c}}$, "0" - the axial magnetic fields being parallel). RL0 belongs to the bounce interaction which has very little reconnection, the flux tubes bounce off each other, and negligible magnetic energy is released from reconnection (Linton et al. 2001). The inflating OL in Figs. 3a, b and the distinct interface between the EFR and the OL in Figs. $4 \mathrm{a}, \mathrm{b}$ are possible evidence that bounce interactions take place. If so, where does the energy for the flare and the EUV brightening event come from?

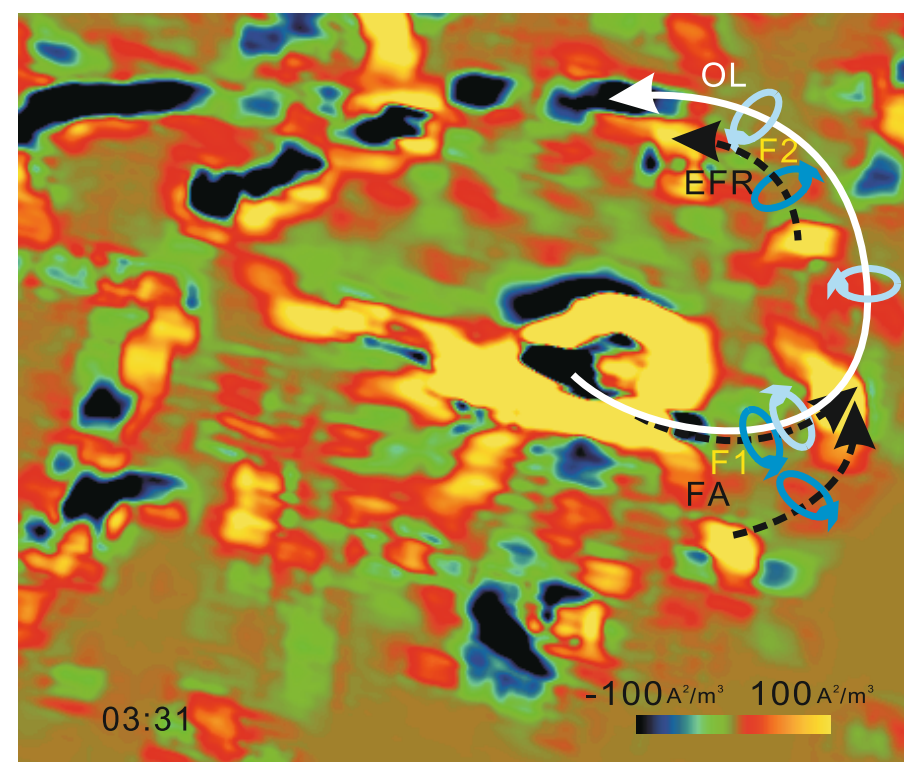

Fig. 5. "F1", "F2" counter-helicity interactions between the flaring arcades (FA) and the overlying loop (OL) and between the emerging flux region (EFR) and the OL. Background is the contour of current helicity measured by Wang et al. (2004). Arrowheads indicate the directions of axial and azimuthal magnetic fields. The situations shown in the sketch are very similar to the RL0 case in Linton et al. (2001).

Moon et al. (2002) found that there is a positive correlation between the impulsively injected magnetic helicity and the X-ray peak flux of the associated flare. Kusano et al. (2003, 2004) then suggested that the sign-reversal of the helicity injection is crucial for the flare/CME activity. Nindos \& Andrews (2004) report that the amount of the stored preflare coronal helicity may determine whether a large flare will be eruptive or confined. Labonte et al. (2007) also found that the necessary condition for an X-flare is that the peak helicity flux has a high magnitude. However, using numerical simulations of the CME breakout model, Phillips et al. (2005) showed that eruption occurs at a fixed magnitude of free energy in the corona, independent of the value of helicity. Possibly, as analyzed by Linton et al. (2001), another important factor, the flux tubes' contact angle, should not be ignored. Linton et al. (2001) also discussed the case of RR0 which belongs to the merge interactions. In such interactions, there is significant reconnection involving mostly the azimuthal field and the two interacting flux tubes merge into one. Energy is released because of a reduction in azimuthal magnetic energy (Linton et al. 2001). As shown in Figs. 2a, b, 3a-c and 4, the FA and the OL are both composed of many single flux fibres. When the RL0 bounce interactions take place, these inner flux fibres will collide with each other with a tensile force from the RL0 interfaces, and finally fall into RR0 or LL0 merge interactions. Therefore we suggest that the solar events are ultimately triggered by Linton's merge interactions. The simplifications of FA and OL found in the southwestern corners of Figs. $3 \mathrm{c}$ and $4 \mathrm{~d}$ confirm our suggestion. Moreover, the unbrightened interface in Figs. $4 a, b$ shows that the energy is released from the interiors of OL and EFR, not from their interface.

At present, we have examined 29 ARs. Among them only AR 8375 can be studied without ambiguity. The other ARs are not suitable for two reasons. (1) It is difficult to discern accurately the positions of coronal loop foot points on the photosphere; (2) there are few available measurements of vector magnetic fields. We believe the STEREO and HINODE missions 
can greatly reduce such two observational limits during solar cycle 24 .

Acknowledgements. This work is jointly supported by the National Natural Science Foundation of China (40621003, 40604019, 40504020, and 40536029), the 973 project under grant 2006CB806304, and the CAS International Partnership Program for Creative Research Teams.

\section{References}

Kusano, K., Yokoyama, T., Maeshiro, T., et al. 2003, Adv. Space Res., 32, 1931 Kusano, K., Maeshiro, T., Yokoyama, T., et al. 2004, ApJ, 610, 537
Labonte, B. J., Georgoulis, M. K., \& Rust, D. M. 2007, ApJ, 671, L955 Linton, M. G., Dahlburg, R. B., \& Antiochos, S. K. 2001, ApJ, 553, 905 Moon, Y.-J., Chae, J., Wang, H. M., et al. 2002, ApJ, 580, 528

Moon, Y.-J., Chae, J., Choe, G. S., et al. 2004, JKAS, 37, 41

Nindos, A., \& Andrews, M. D. 2004, ApJ, 616, L175

Nitta, N. V., \& Hudson, H. S. 2001, GRL, 28, 3801

Phillips, A. D., Macneice, P. J., \& Antiochos, S. K. 2005, ApJ, 624, L129 Pohjolainen, S. 2003, Sol. Phys., 213, 319

Romano, P., Contarino, L., \& Zuccarello, F. 2003, Sol. Phys., 218, 137

Wang, J. X., Zhou, G. P., \& Zhang, J. 2004, ApJ, 615, 1021

Yurchyshyn, V. B., \& Wang, H. 2001, Sol. Phys., 202, 309

Zhang, J., \& Wang, J. X. 2002, ApJ, 566, L117 